\title{
Decreased cardiac mitochondrial tetrahydrobiopterin in a rat model of pressure overload
}

\author{
SHUNICHI SHIMIZU, MASAAKI ISHIBASHI, SUMITO KUMAGAI, TERUAKI WAJIMA, \\ TOSHIHITO HIROI, TATSUYA KURIHARA, MASAKAZU ISHII and YUJI KIUCHI
}

Department of Pathophysiology, Showa University School of Pharmacy, Shinagawa-ku, Tokyo 142-8555, Japan

Received October 25, 2012; Accepted December 19, 2012

DOI: 10.3892/ijmm.2013.1236

\begin{abstract}
Sustained cardiac pressure overload induces mitochondrial dysfunction and apoptosis of cardiomyocytes leading to pathological cardiac hypertrophy and dysfunction. Mitochondrial nitric oxide synthase (NOS) appears to cause uncoupling, which produces reactive oxygen species (ROS) instead of nitric oxide (NO), by a decrease in the cofactor tetrahydrobiopterin (BH4). This study focused on examining the changes in mitochondrial BH4 levels during cardiac pressure overload. Chronic cardiac pressure overload was generated by abdominal aortic banding in rats. Levels of $\mathrm{BH} 4$ and its oxidized form were measured in the mitochondria isolated from the left ventricle (LV) and the post-mitochondrial supernatants. Chronic aortic banding increased blood pressure, and induced cardiac hypertrophy and fibrosis. Notably, the BH4 levels were decreased while its oxidized forms were increased in LV mitochondria, but not in the post-mitochondrial supernatants containing the cytosol and microsome. Anti-neuronal NOS antibody-sensitive protein was detected in the cardiac mitochondria. Moreover, continuous administration of $\mathrm{BH} 4$ to rats with pressure overload increased mitochondrial $\mathrm{BH} 4$ levels and reduced cardiac fibrosis and matrix metallopeptidase activity, but not cardiac hypertrophy. These findings show the possibility that NOS uncoupling by decreased cardiac mitochondrial $\mathrm{BH} 4$ levels is implicated, at least in part, in the development of cardiac fibrosis, leading to cardiac dysfunction induced by pressure overload.
\end{abstract}

\section{Introduction}

Left ventricular hypertrophy represents an adaptive mechanism through which the heart normalizes ventricular wall

Correspondence to: Dr Shunichi Shimizu, Department of Pathophysiology, Showa University School of Pharmacy, 1-5-8 Hatanodai, Shinagawa-ku, Tokyo 142-8555, Japan

E-mail:shun@pharm.showa-u.ac.jp

Key words: cardiac pressure overload, mitochondria, tetrahydrobiopterin, nitric oxide synthase stress and preserves systolic function in the early stages; however, sustained hypertrophic stimulation frequently leads to contractile dysfunction of the myocardium through myocardial cell damage and cardiac fibrosis, and worsens the risk of morbidity and mortality due to congestive heart failure and sudden death. Accumulating evidence shows the implication of reactive oxygen species (ROS) in all processes causing terminal cardiac damage: cardiac hypertrophy, myocardial cell death and cardiac fibrosis $(1,2)$.

Nitric oxide synthase (NOS) is an enzyme that produces nitric oxide (NO) from L-arginine and molecular oxygen, and three mammalian isoforms have been identified, including neuronal NOS (nNOS or NOS1), inducible NOS (iNOS or NOS2) and endothelial NOS (eNOS or NOS3). Tetrahydrobiopterin (BH4), a naturally occurring potent reducing agent, is an essential cofactor for all three isoforms of NOS (3-6). All isoforms of NOS are only activated as homodimers, and the stabilization and maintenance of the NOS dimer are dependent on BH4 (7). BH4 also plays a crucial role as an electron donor in the multistep oxidation of arginine for the generation of NO. Importantly, a decrease in BH4 leads to the uncoupling of NOS, resulting in the reduced production of NO and increased production of ROS (8-10). The simultaneous release of NO and ROS from NOS forms peroxynitrite $\left(\mathrm{ONOO}^{-}\right)$, a strong oxidant. Malo et al (11) reported that treatment with $\mathrm{BH} 4$ prevented endothelial dysfunction in epicardial coronary arteries associated with left ventricular hypertrophy in a porcine model. Moreover, administration of BH4 has been shown to protect the myocardium against pressure overload, resulting in the improvement of fibrosis, cardiac dysfunction and hypertrophy (12-14). Takimoto et al (15) showed that pressure overload triggers NOS3 uncoupling as a prominent source of myocardial ROS contributing to dilatory remodeling and cardiac dysfunction using NOS3-deficient mice. NOS3 is the dominant isoform in the vascular endothelium as well as in cardiac myocytes. Thus, uncoupling of NOS3 by decreased BH4 appears to mediate cardiac hypertrophy and remodeling by pressure overload. Interestingly, accumulated evidence has shown the presence of NOS in mitochondria (16-18). The mitochondrial NOS isoenzyme is a constitutive protein of the mitochondrial inner membrane that generates $\mathrm{NO}$ in a $\mathrm{Ca}^{2+}$-dependent reaction (19). Although the role of NO in mitochondria remains to be elucidated, mitochondrial events such as 
oxygen consumption and ROS production appear to be regulated by NO $(17,20,21)$. Notably, mitochondrial NOS also seems to cause uncoupling (22). These observations allow us to hypothesize that uncoupling of mitochondrial NOS by decreased $\mathrm{BH} 4$ during pressure overload is implicated in mitochondrial damage and results in myocardial cell damage, leading to cardiac hypertrophy and/or cardiac fibrosis. In fact, it has been demonstrated that pressure overload-induced heart failure is associated with mitochondrial dysfunction (23-26); however, the changes in BH4 levels in cardiac mitochondria during pressure overload remain to be elucidated.

In the present study, we firstly examined changes in $\mathrm{BH} 4$ content in mitochondria and post-mitochondrial supernatant during pressure overload, and found that $\mathrm{BH} 4$ levels were decreased in cardiac mitochondria; therefore, we next examined whether administration of $\mathrm{BH} 4$ to rats with pressure overload increases the mitochondrial BH4 level and improves cardiac hypertrophy and/or cardiac fibrosis.

\section{Materials and methods}

Study approval and ethics. The animals used in this study were handled in accordance with the Guide for the Care and Use of Laboratory Animals published by the US National Institute of Health (NIH publication no. 85-23, revised 1996), and the protocol was approved by the Experimental Animal Committee of Showa University (\#20050).

Animals and preparation. Male Sprague-Dawley rats (9 weeks old) were housed in a humidity- and temperaturecontrolled environment with an automatic 12:12-h light-dark cycle and were fed standard rat chow and tap water ad libitum. Rats were anesthetized with sodium pentobarbital $(50 \mathrm{mg} / \mathrm{kg}$, i.p.). A rat model of pressure overload was prepared according to a previously described method (27). The abdominal aorta was constricted between the right and left renal arteries, using a 23-gauge needle to establish the diameter of the ligature. Sham-operated rats underwent an identical procedure except for the ligature. BH4 $(10 \mathrm{mg} / \mathrm{kg} / \mathrm{day})$ was administered

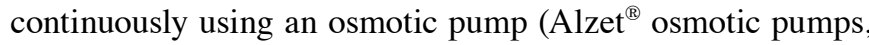
2ML4; LMS Co., Tokyo, Japan). Although BH4 is unstable in saline, ascorbate is known to stabilize it (28-30); therefore, $1 \mathrm{mM}$ ascorbate was used as a stabilizing reagent. An osmotic pump filled with saline, ascorbate or ascorbate plus BH4 was placed in a subcutaneous area in the back. One or 4 weeks after surgery, rats were again anesthetized with sodium pentobarbital $(50 \mathrm{mg} / \mathrm{kg}$, i.p.). The right carotid artery was cannulated for measurement of arterial blood pressure and heart rate. The heart was then excised in ice-cold phosphatebuffered saline and the ventricles were divided into left and right ventricles.

Isolation of cardiac mitochondria and post-mitochondrial supernatant. Cardiac mitochondria were isolated as described previously (31). The isolated left ventricle (LV) was immediately minced and homogenized by a Teflon Potter-Elvejhem homogenizer in MST solution containing $0.23 \mathrm{M}$ mannitol, $0.07 \mathrm{M}$ sucrose, $1 \mathrm{mM}$ EDTA and $10 \mathrm{mM}$ Tris- $\mathrm{HCl}(\mathrm{pH}$ 7.4). The homogenate was centrifuged at $700 \mathrm{x}$ g for $10 \mathrm{~min}$, and the supernatant was collected and centrifuged at $8,000 \mathrm{x}$ g for
$10 \mathrm{~min}$. The mitochondrial pellet and the post-mitochondrial supernatant containing the cytosol and microsome were collected. The mitochondrial pellet was washed once with MST solution.

Measurement of biopterin derivatives. Biopterin derivatives were measured as biopterin by differential oxidation as described previously (32). An aliquot of the ventricular homogenate, mitochondria or the post-mitochondrial supernatant was separately oxidized in an acidic condition $(0.02 \mathrm{M}$ $\mathrm{KI} / \mathrm{I}_{2}$ in $\left.0.1 \mathrm{M} \mathrm{HCl}\right)$ and a base condition $\left(0.02 \mathrm{M} \mathrm{KI} / \mathrm{I}_{2}\right.$ in $0.1 \mathrm{M} \mathrm{NaOH}$ ). Quantification of biopterin was performed by reverse-phase high performance liquid chromatography with fluorometric detection (33). The amount of BH4 was calculated from the difference in the biopterin concentrations measured after oxidation in the acid (total biopterin) and base [7,8-dihydrobiopterin (BH2) plus biopterin].

Measurement of $m R N A$ levels of NOSs by reverse transcriptase (RT)-PCR. Total RNA was extracted from the heart using a modified guanidinium isothiocyanate method with TRIzol ${ }^{\circledR}$ reagent (Invitrogen, Tokyo, Japan). RT-PCR analysis of NOS1, NOS2 and NOS3 mRNAs was performed as previously described (34). Reverse transcription and PCR amplification from $0.2 \mu \mathrm{g}$ total RNA were performed using rTth DNA polymerase (RT-PCR High Plus ${ }^{\circledR}$; Toyobo Co., Osaka, Japan). The pairs of primers used included: NOS1 (NM_052799), 5'-GACCCACGTGGTCCTCATTC-3' and 5'-CCTGGATTCC TGTGTCTTTC-3'; NOS2 (NM_012611), 5'-CGCTACACTTC CAACGCAAC-3' and 5'-AGGAAGTAGGTGAGGGCTTG-3'; NOS3 (NM_02183), 5'-CTAGACACCCGGACAACC-3' and 5'-GCTGCTGTGCGTAGCTCT-3', respectively. PCR products were electrophoresed on $3 \%$ agarose gel containing ethidium bromide and visualized by UV-induced fluorescence.

Western blot analysis. The obtained mitochondria, brain and aorta were lysed in lysis buffer containing $50 \mathrm{mM}$ Tris- $\mathrm{HCl}$ (pH 7.4), 1\% NP-40, $0.25 \%$ deoxycholic acid, $15 \mathrm{mM} \mathrm{NaCl}, 0.1 \mathrm{mM}$ EDTA, $1 \mathrm{mM}$ PMSF, $1 \mathrm{mM}$ sodium orthovanadate, $1 \mathrm{mM} \mathrm{NaF}$ and $1 \mu \mathrm{g} / \mathrm{ml}$ each of aprotinin, leupeptin and pepstatin, and then treated with ultrasonication for $5 \mathrm{sec}$. The lysates were centrifuged at $15,000 \mathrm{x}$ g for $10 \mathrm{~min}$ at $4^{\circ} \mathrm{C}$. After the supernatants were collected, the protein concentration was determined using the DC Protein Assay Kit ${ }^{\circledR}$ (Bio-Rad Laboratories, Hercules, CA, USA). Samples containing equal amounts of protein $(40 \mu \mathrm{g})$ were separated on $10 \%$ SDS-polyacrylamide gels under reducing conditions and transferred onto Hybond ECL ${ }^{\circledR}$ nitrocellulose membranes (GE Healthcare, Tokyo, Japan). Nonspecific binding was blocked with $5 \% \mathrm{ECL}^{\circledR}$ blocking agent (GE Healthcare) in TBS containing $0.1 \%$ Tween-20 (TBS-T) for $60 \mathrm{~min}$. The membranes were incubated overnight at $4^{\circ} \mathrm{C}$ with a 1:5,000 dilution of rabbit anti-nNOS antibody (SigmaAldrich, Tokyo, Japan), a 1:1,000 dilution of rabbit anti-iNOS antibody (Santa Cruz Biotechnology, Inc., Santa Cruz, CA, USA), a 1:1,000 dilution of goat anti-NOS3 antibody (Santa Cruz Biotechnology, Inc.), a 1:2,000 dilution of mouse anti-cytochrome $c$ antibody (R\&D Systems, Minneapolis, MN, USA) or a 1:2,000 dilution of goat anti-GAPDH antibody (Santa Cruz Biotechnology, Inc.) and developed with 
Table I. Changes in hemodynamic parameters and heart weight by aortic banding.

\begin{tabular}{|c|c|c|}
\hline & $\begin{array}{l}\text { Sham } \\
(n=7)\end{array}$ & $\begin{array}{l}\text { Aortic banding } \\
\qquad(\mathrm{n}=7)\end{array}$ \\
\hline \multicolumn{3}{|c|}{ Body weight (g) } \\
\hline 1 week & $329.9 \pm 4.4$ & $285.4 \pm 11.7^{\mathrm{a}}$ \\
\hline 4 weeks & $397.3 \pm 5.8$ & $356.7 \pm 10.9^{\mathrm{a}}$ \\
\hline \multicolumn{3}{|c|}{ Heart weight (g) } \\
\hline 1 week & $0.78 \pm 0.01$ & $0.98 \pm 0.03^{\mathrm{a}}$ \\
\hline 4 weeks & $0.94 \pm 0.04$ & $1.26 \pm 0.08^{\mathrm{a}}$ \\
\hline \multicolumn{3}{|c|}{ Heart weight (mg)/body weight (g) } \\
\hline 1 week & $2.37 \pm 0.05$ & $3.48 \pm 0.18^{\mathrm{a}}$ \\
\hline 4 weeks & $2.36 \pm 0.10$ & $3.53 \pm 0.18^{\mathrm{a}}$ \\
\hline \multicolumn{3}{|c|}{ LV weight (g) } \\
\hline 1 week & $0.61 \pm 0.01$ & $0.80 \pm 0.03^{\mathrm{a}}$ \\
\hline 4 weeks & $0.74 \pm 0.03$ & $1.04 \pm 0.05^{\mathrm{a}}$ \\
\hline \multicolumn{3}{|c|}{ LV weight (mg)/body weight (g) } \\
\hline 1 week & $1.85 \pm 0.04$ & $2.82 \pm 0.15^{\mathrm{a}}$ \\
\hline 4 weeks & $1.87 \pm 0.08$ & $2.91 \pm 0.11^{\mathrm{a}}$ \\
\hline \multicolumn{3}{|c|}{ Mean blood pressure (mmHg) } \\
\hline 1 week & $107.6 \pm 4.3$ & $158.0 \pm 6.6^{\mathrm{a}}$ \\
\hline 4 weeks & $104.7 \pm 4.4$ & $153.3 \pm 7.4^{\mathrm{a}}$ \\
\hline \multicolumn{3}{|c|}{ Systolic blood pressure (mmHg) } \\
\hline 1 week & $125.0 \pm 5.8$ & $180.4 \pm 8.6^{\mathrm{a}}$ \\
\hline 4 weeks & $118.3 \pm 5.4$ & $167.9 \pm 11.2^{\mathrm{a}}$ \\
\hline \multicolumn{3}{|c|}{ Diastolic blood pressure (mmHg) } \\
\hline 1 week & $87.4 \pm 3.6$ & $136.5 \pm 5.7^{\mathrm{a}}$ \\
\hline 4 weeks & $90.7 \pm 3.8$ & $138.5 \pm 8.0^{\mathrm{a}}$ \\
\hline \multicolumn{3}{|c|}{ Heart rate (bmp) } \\
\hline 1 week & $376.5 \pm 14.5$ & $366.1 \pm 21.9$ \\
\hline 4 weeks & $374.3 \pm 7.8$ & $358.7 \pm 20.5$ \\
\hline
\end{tabular}

Values indicate the average \pm SEM of 7 rats. ${ }^{a} \mathrm{P}<0.05$, significantly different from each sham-operated group.

an enhanced chemiluminescence western blotting detection system (GE Healthcare) with a horseradish peroxidase (P)-conjugated secondary antibody. As the secondary antibody, a 1:2,000 dilution of P-conjugated anti-rabbit IgG (Cell Signaling Technology, Inc., Tokyo, Japan), a 1:20,000 dilution of P-conjugated anti-goat IgG (Jackson ImmunoResearch, West Grove, PA, USA) or a 1:10,000 dilution of P-conjugated anti-mouse IgG was used. The membranes were exposed to chemiluminescence-sensitive film (GE Healthcare) for 1-3 $\min$.

Zymography. Matrix metallopeptidase (MMP) activities were measured by gelatin zymography. The isolated LV was minced and homogenized by a Polytron homogenizer in lysis buffer containing $50 \mathrm{mM}$ Tris- $\mathrm{HCl}$ (pH 7.4), 1\% NP-40, $0.25 \%$ deoxycholic acid, $15 \mathrm{mM} \mathrm{NaCl}, 0.1 \mathrm{mM}$ EDTA, $1 \mathrm{mM}$ PMSF, and $1 \mu \mathrm{g} / \mathrm{ml}$ each of aprotinin, leupeptin and pepstatin.
The samples were then sonicated for $5 \mathrm{sec}$ on ice and centrifuged at $15,000 \mathrm{x} \mathrm{g}$ for $15 \mathrm{~min}$. After the supernatants were collected, the protein concentration was determined using the DC Protein Assay Kit. Zymography was performed using a gelatin zymography kit (Primary Cell Co., Ltd., Hokkaido, Japan).

Histological analysis. Hearts were fixed with $10 \%$ formamide. The fixed heart was embedded in paraffin, sectioned at a 4- $\mu \mathrm{m}$ thickness, and stained by Giemsa for fibrosis. Fibrosis was scored as $0-3$ by individuals blinded to the study protocol.

Statistical analysis. All values are expressed as the means \pm SEM. Statistical analyses of the data were carried out using the unpaired Student's t-test or ANOVA followed by Bonferroni test, as appropriate. The level of statistical significance was set at $\mathrm{P}<0.05$.

\section{Results}

Hemodynamic parameters and heart weights in rats with aortic banding. Hemodynamic parameters and heart weights at 1 and 4 weeks after the sham operation or aortic banding are summarized in Table I. The mean carotid arterial, systolic and diastolic pressure were significantly increased by aortic banding compared to each sham-operated group. The heart rate was not significantly different between groups. Thus, pressure overload on the LV was observed in this aortic banding model. The body weight of rats was slightly decreased after aortic banding. The heart weights at 1 week $(0.78 \pm 0.01 \mathrm{~g})$ and 4 weeks $(0.94 \pm 0.04 \mathrm{~g})$ after the sham operation were significantly increased to $0.98 \pm 0.03$ and $1.26 \pm 0.08 \mathrm{~g}$ by aortic banding, respectively. The heart weight to body weight ratios markedly increased from $2.37 \pm 0.05$ to $3.48 \pm 0.18$ ( 1 week) and from $2.36 \pm 0.10$ to $3.55 \pm 0.18$ (4 weeks) by aortic banding, respectively. The $\mathrm{LV}$ weight and $\mathrm{LV}$ weight to body weight ratios were also increased after aortic banding compared to each sham-operated group.

Decrease in mitochondrial BH4 levels by pressure overload. We firstly measured $\mathrm{BH} 4$ levels in the left and right ventricles at 1 and 4 weeks after pressure overload. BH4 levels in the left and right ventricles at 1 week post-pressure overload were not altered when compared to each sham-operated group (Fig. 1A). Although a statistical difference was not reached, BH4 levels in the LV but not in the right ventricle (RV) at 4 weeks post-pressure overload slightly tended to decrease (Fig. 1B). To examine the changes in $\mathrm{BH} 4$ levels in the myocardium in detail, we next measured the levels of BH4 and its oxidized forms in the mitochondria and the post-mitochondrial supernatant isolated from LVs. One week post-pressure overload, BH4 and its oxidized form levels in either mitochondria or the post-mitochondrial supernatant were not significantly altered when compared to each sham-operated group (Fig. 2A). The ratios of $\mathrm{BH} 4$ level to oxidized biopterin level in the mitochondria $(3.11 \pm 0.29)$ and the post-mitochondrial supernatant $(11.60 \pm 0.62)$ were also not decreased (mitochondria: $2.47 \pm 0.17$, post-mitochondrial supernatant: $10.12 \pm 1.13$ ). Notably, 4 weeks post-pressure overload, the mitochondrial $\mathrm{BH} 4$ levels were 

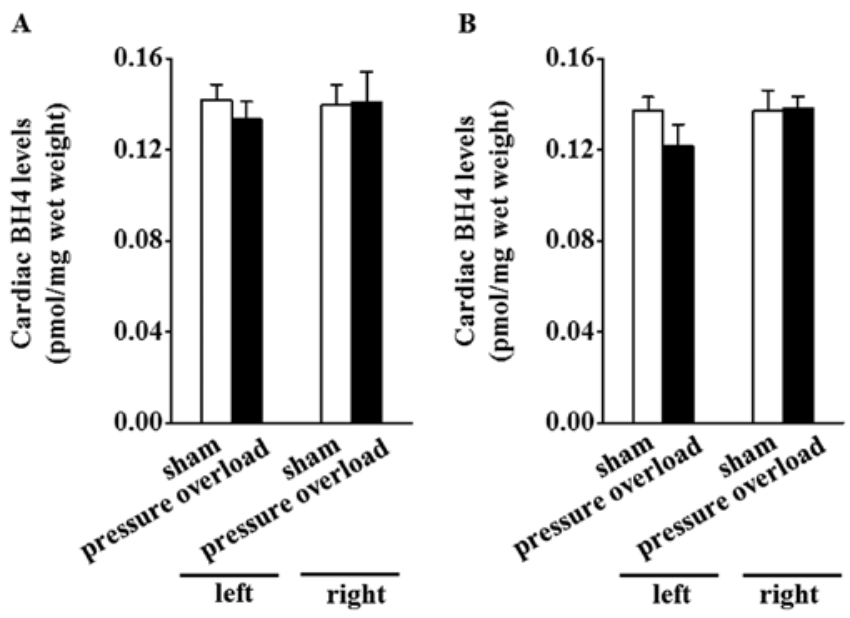

Figure 1. Cardiac BH4 levels in response to pressure overload. BH4 levels in the left and right ventricles were determined at (A) 1 week and (B) 4 weeks after pressure overload. Open bars indicate sham group; closed bars indicate the pressure overload group. Values represent the average \pm SEM of 5 animals.
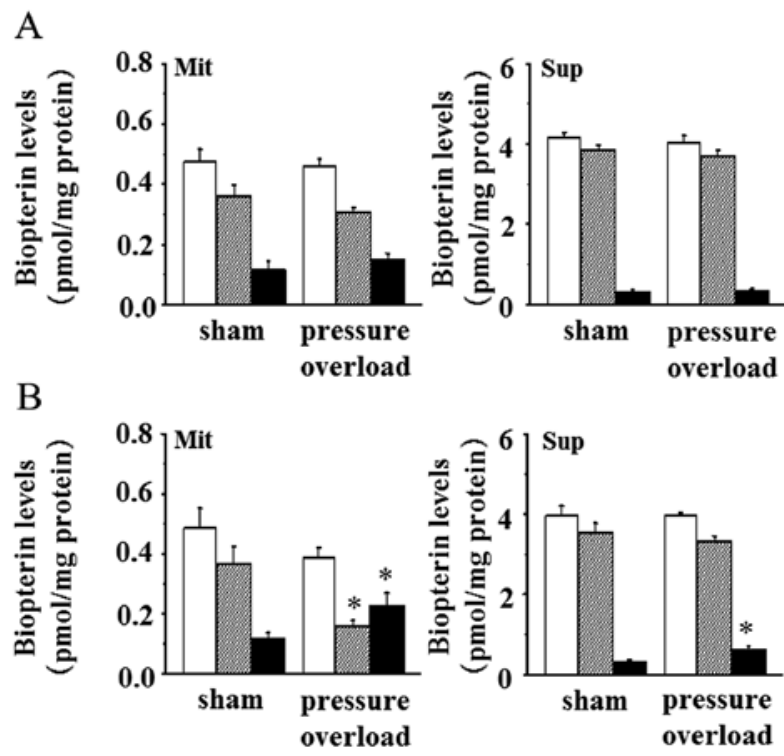

$\mathrm{C}$

Mit Sup

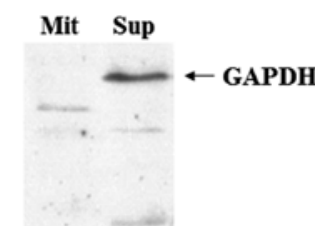

Figure 2. Changes in BH4 levels in cardiac mitochondria and the post-mitochondrial supernatant by pressure overload. The hearts were isolated from sham-operated rats and pressure overload rats at (A) 1 week or (B) 4 weeks after pressure overload. The mitochondrial fraction (Mit) and the postmitochondrial supernatant fraction (Sup) were prepared from the isolated heart, and the levels of total biopterin (open column), BH4 (striped column) and its oxidized form (closed column) were determined. Values represent the average \pm SEM of 5 animals. ${ }^{*} \mathrm{P}<0.05$, significantly different from each sham-operated group. (C) Proteins extracted from mitochondria and the post-mitochondrial supernatants were separated by polyacrylamide gel, and cytochrome $c$ and GAPDH were detected using western blotting.

significantly decreased, and its oxidized forms were increased (Fig. 2B, right panel). In the post-mitochondrial supernatant, the BH4 levels were not altered, while its oxidized forms
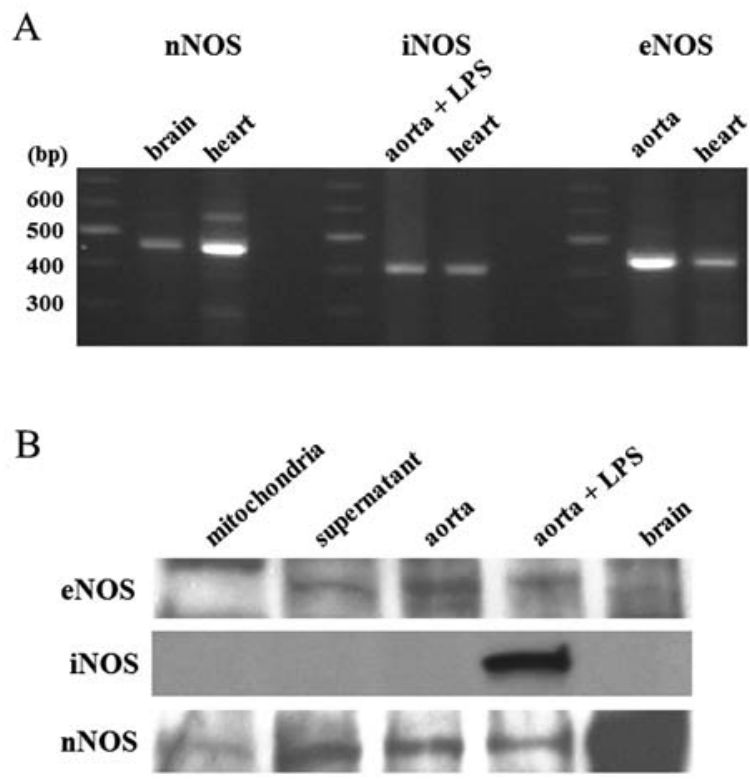

Figure 3. Expression of NOS isoforms in the heart. (A) Total RNA was extracted from the heart, and the expression of NOS isoform mRNAs was measured by RT-RCR. As a positive control, total RNA was extracted from the aorta (NOS3), the aorta treated with $10 \mathrm{ng} / \mathrm{ml}$ LPS for $12 \mathrm{~h}$ (NOS2) and the brain (NOS1), and parallel RT-PCR was performed. Theoretical product sizes of NOS1, NOS2 and NOS3 were 433, 406 and 409 bp, respectively. (B) Proteins were extracted from the heart, and the expression of NOS isoform proteins was measured by western blotting. As a positive control, proteins from the aorta (eNOS), the aorta treated with $10 \mathrm{ng} / \mathrm{ml}$ LPS for $12 \mathrm{~h}$ (NOS2) and the brain (nNOS) were used.

were increased (Fig. 2B, left panel). The ratios of BH4 level to oxidized biopterin level in the mitochondria $(3.13 \pm 0.32)$ and in the post-mitochondrial supernatant $(11.33 \pm 1.58)$ markedly decreased to $0.70 \pm 0.08(\mathrm{P}<0.05)$ and $3.33 \pm 0.24(\mathrm{P}<0.05)$, respectively.

To confirm whether mitochondria and the post-mitochondrial supernatant were separated, the markers for mitochondria and cytosol were measured by western blot analysis (Fig. 2C). Cytochrome $c$, a marker for mitochondria, was detected in the mitochondrial fraction, but not in the postmitochondrial supernatant. In contrast, GAPDH, a marker for cytosol, was observed in the post-mitochondrial supernatant, but not in the mitochondrial fraction; thus, mitochondria and the post-mitochondrial supernatant were separated in our experimental condition.

Expression of NOS isoforms in the myocardium, cardiac mitochondria and the post-mitochondrial supernatant. The expression of NOS isoform mRNAs in the heart was measured by RT-PCR. As shown in Fig. 3A, all 3 isoforms of NOS were observed in the heart. Therefore, we next measured NOS isoform protein in the mitochondria and the post-mitochondrial supernatant (Fig. 3B). Anti-NOS3 antibody-sensitive protein was detected in the post-mitochondrial supernatant and the aorta (positive control), but not in the mitochondria. Although anti-iNOS antibody-sensitive protein was detected in the LPS-treated aorta (positive control), it was not observed in either the mitochondria or the postmitochondrial supernatant. Anti-NOS1 antibody-sensitive 
A

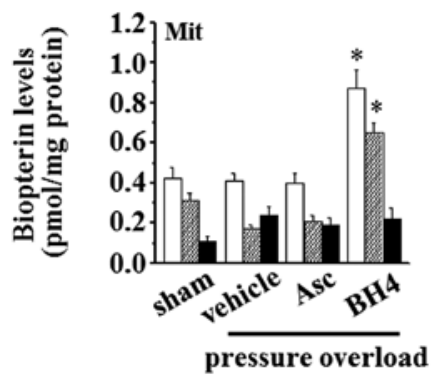

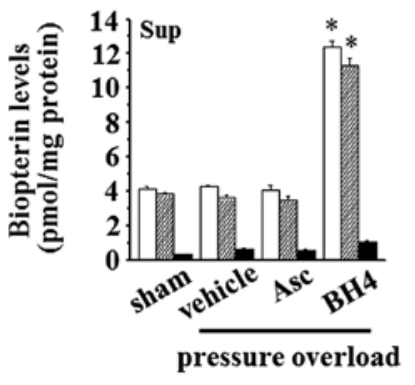

B

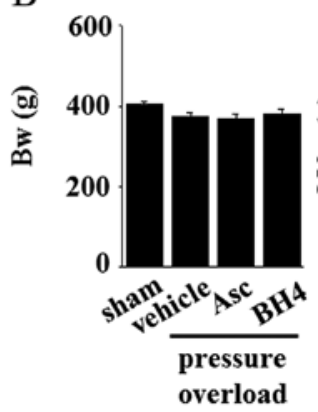

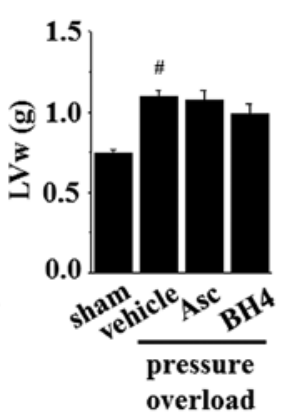

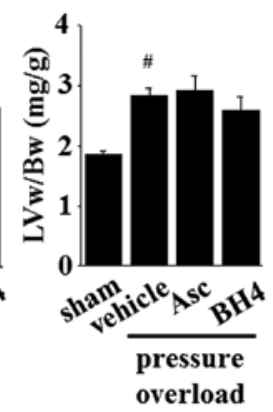

Figure 4. Effect of the administration of BH4 on the levels of BH4 and its oxidized form in cardiac mitochondria and the post-mitochondrial supernatant, and on left ventricular weight. Rats were treated with sham operation, pressure overload, pressure overload with ascorbate (Asc) administration or pressure overload with ascorbate plus BH4 for 4 weeks. (A) Levels of total biopterin (open column), BH4 (striped column) and its oxidized form (closed column) in the left ventricle (LV) were determined. (B) Body weight (Bw) and LV weight (LVw) were measured, and the LV weight to body weight ratio (LVw/Bw) was calculated. Values represent the average \pm SEM of 5 animals. ${ }^{*} \mathrm{P}<0.05$, significantly different from pressure overload-alone group. ${ }^{\sharp} \mathrm{P}<0.05$, significantly different from sham-operated group.

A

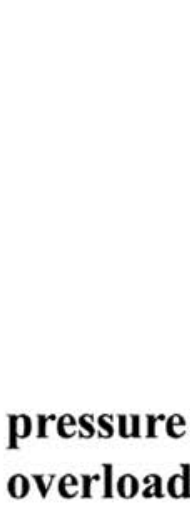

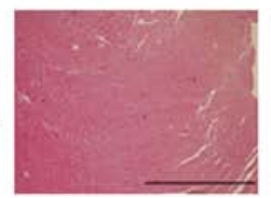

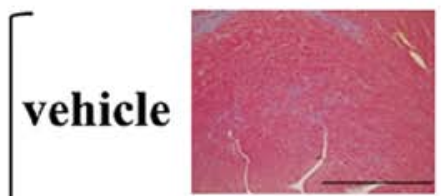

Asc

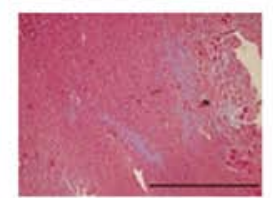

BH4

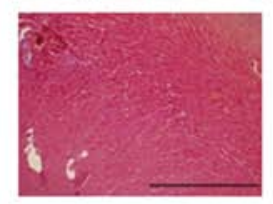

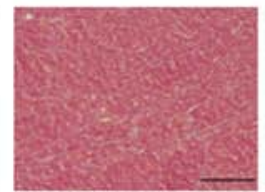
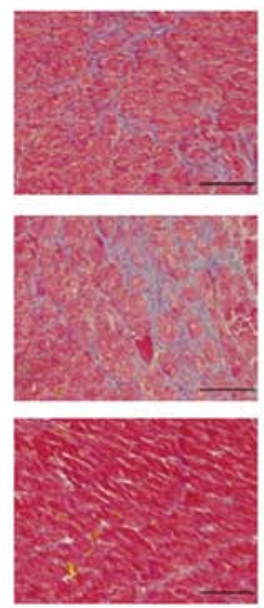

B

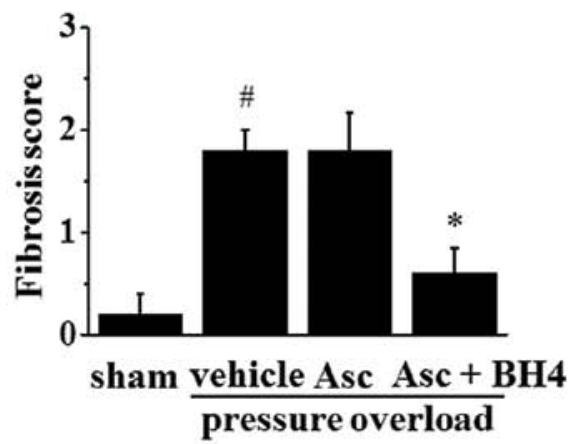

Figure 5. Effect of BH4 administration on pressure overload-induced cardiac fibrosis. Rats were treated with sham operation, pressure overload, pressure overload with ascorbate (Asc) administration or pressure overload with ascorbate plus BH4 for 4 weeks. (A) Typical images of LV tissues with Giemsa staining. Scale bars in right and left panels indicate $2 \mathrm{~mm}$ and $200 \mu \mathrm{m}$, respectively. (B) The fibrosis scores in each group were calculated. Values represent the average \pm SEM of 5 animals. ${ }^{~} \mathrm{P}<0.05$, significantly different from sham-operated group. ${ }^{*} \mathrm{P}<0.05$, significantly different from pressure overload-alone group.

protein was detected in mitochondria and the brain (positive control), but not in the post-mitochondrial supernatant. Thus, anti-NOS1 antibody-sensitive protein was localized in the cardiac mitochondria.

Effect of BH4 administration on cardiac hypertrophy. We next examined the effect of continuous administration of $\mathrm{BH} 4$ on hypertrophy 4 weeks following pressure overload. Ascorbate was used to stabilize BH4. Fig. 4A shows the levels of BH4 and its oxidized form in the mitochondria and the post-mitochondrial supernatant. BH4 levels in the mitochondria and the post-mitochondrial supernatant were significantly increased by administration of BH4; however, cardiac hypertrophy was not improved by administration of BH4 (Fig. 4B). The mean carotid arterial pressure in the pressure overload rats $(163.5 \pm 15.4 \mathrm{mmHg}$, $\mathrm{n}=5$ ) was not significantly altered by the administration of ascorbate $(174.9 \pm 25.7 \mathrm{mmHg}, \mathrm{n}=5)$ or ascorbate plus $\mathrm{BH} 4$ $(151.5 \pm 17.9 \mathrm{mmHg}, \mathrm{n}=5)$.

Effects of BH4 administration on cardiac fibrosis and MMP activity. Cardiac fibrosis was measured by Giemsa staining and the fibrosis was scored (Fig. 5B). Typical images are shown in Fig. 5A. Interstitial fibrosis was observed 4 weeks following pressure overload, and fibrosis was prevented by the administration of BH4, but not ascorbate (Fig. 5B). Moreover, cardiac MMP activity, an important factor in the process of fibrosis, was measured 4 weeks following pressure overload 
A
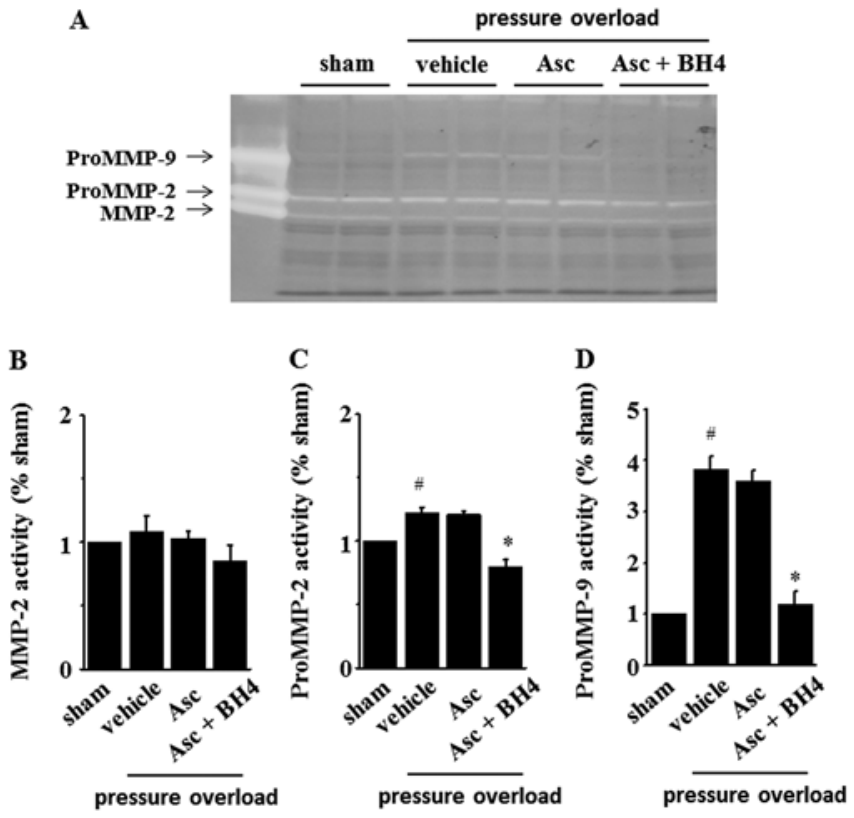

Figure 6. Effect of BH4 administration on MMP activity in cardiac tissue induced by pressure overload. Rats were treated with sham operation, pressure overload, pressure overload with ascorbate (Asc) administration or pressure overload with ascorbate plus BH4 for 4 weeks. (A) Typical zymography for MMP-2, proMMP-2 and proMMP-9. (B) The activity of each MMP was calculated from the density of the zymography. ${ }^{\#} \mathrm{P}<0.05$, significantly different from sham-operated group. ${ }^{*} \mathrm{P}<0.05$, significantly different from pressure overload-alone group.

(Fig. 6). ProMMP-2 and ProMMP-9 activity were increased, and the increase was reduced by the administration of $\mathrm{BH} 4$, but not ascorbate (Fig. 6A and B). MMP-2 activity was not significantly altered.

\section{Discussion}

In a rat model of abdominal aortic pressure overload, we found that the $\mathrm{BH} 4$ levels and the ratio of $\mathrm{BH} 4$ to oxidized biopterin ratio markedly decreased in the cardiac mitochondria. Anti-NOS1 antibody-sensitive protein was observed in cardiac mitochondria, which is consistent with a previous report (19). Moreover, continuous administration of $\mathrm{BH} 4$ increased mitochondrial BH4 levels, and prevented cardiac fibrosis and MMP activity. These findings suggest that NOS uncoupling by decreased $\mathrm{BH} 4$ in cardiac mitochondria is implicated in the development of cardiac fibrosis induced by pressure overload.

We observed anti-NOS1 antibody-sensitive protein in cardiac mitochondria. It has been shown that NOS is located in mitochondria $(16,17)$. Zaobornyj et al (35) reported that $\mathrm{NO}$ is produced in the myocardium in physiologically relevant quantities by two isoforms of NOS: i) an isoenzyme located in the mitochondria, which accounts for $\sim 55-62 \%$ of total heart NO production, and ii) an isoenzyme located in caveolae of the sarcoplasmic reticulum and plasma membrane, which accounts for the remaining $38-45 \%$. Kanai et al (19) reported that NO production was detected from individual mitochondria isolated from the hearts of wild-type, NOS3-knockout and NOS2-knockout, but not from NOS1-knockout mice. NO production was found to be dependent on mitochondrial $\mathrm{Ca}^{2+}$ uptake and was blocked by ruthenium red, indicating that it originated from mitochondria. Moreover, accumulating evidence shows that mitochondrial NOS is mostly a variant of $\operatorname{NOS} 1 \alpha$, which could be subjected to post-transcriptional modification of the product encoded by the NOS1 $\alpha$ gene (18). Thus, a functional active NOS isoform, probably NOS1 or a variant of NOS1 $\alpha$, appears to be located in cardiac mitochondria.

Decreased mitochondrial $\mathrm{BH} 4$ levels may result in cardiac dysfunction and damage. Importantly, a decrease in $\mathrm{BH} 4$ leads to uncoupling of NOS, resulting in the reduced production of NO and the increased production of ROS (8-10). Under physiological conditions, the production of NO by mitochondria has been shown to be important for maintenance of the cellular metabolism, i.e. it modulates oxygen consumption $(17,21,36)$. The decrease in NO production by uncoupling of mitochondrial NOS may lead to mitochondrial dysfunction. Moreover, the increased ROS production by uncoupling of mitochondrial NOS may also lead to mitochondrial dysfunction. Thus, either reduced NO production or increased ROS production from mitochondrial NOS may induce myocardial injury via the dysfunction of mitochondria. Notably, Dedkova and Blatter (22) demonstrated that uncoupling of mitochondrial NOS in cardiomyocytes stimulates mitochondrial ROS production. They concluded the importance of L-arginine and $\mathrm{BH} 4$ for cardioprotection via regulation of mitochondrial oxidative stress and modulation of the mitochondrial permeability transition pore (mPTP) opening by mitochondrial NOS. The opening of mPTP is well known to lead to cell death through the release of apoptogens including cytochrome $c$ from mitochondria to the cytosol $(37,38)$. The report by Dedkova and Blatter (22) allowed us to speculate that uncoupling of mitochondrial NOS may be induced during pressure overload by decreasing BH4 levels in the heart, and the uncoupling of mitochondrial NOS could be related to the induction of cardiac cell death. It is possible that the administration of $\mathrm{BH} 4$ reduces cardiac fibrosis through the prevention of NOS uncoupling-mediated cardiac cell death. In fact, the administration of BH4 during pressure overload reduced cardiac fibrosis, consistent with previous studies $(12,13,15)$. In contrast to cardiac fibrosis, hypertrophy induced by pressure overload was not attenuated by the administration of BH4. Moens et al (12) reported that, when BH4 treatment was started at the onset of pressure overload, it did not suppress hypertrophy over the first week when NOS activity was preserved even in untreated pressure overload hearts. The reason why administration of $\mathrm{BH} 4 \mathrm{did}$ not suppress hypertrophy needs to be examined in a future study.

Takimoto et al (15) found that pressure overload-induced cardiac remodeling and dysfunction were reduced in NOS3deficient mice. As an underlying mechanism, they showed that NOS3 uncoupling is implicated in those responses. We observed that NOS3 was present in the post-mitochondrial supernatant. In cardiomyocytes, NOS3 has been shown to be located in caveolae $(39,40)$. In post-mitochondrial supernatant, BH4 levels decreased, and its oxidized forms increased 4 weeks following pressure overload. This observation 
supports the finding that NOS3 uncoupling with a decrease in $\mathrm{BH} 4$ is implicated in the development of cardiac dysfunction by pressure overload.

In the present study, we focused on changes in BH4 levels induced by pressure overload, and we found that BH4 levels in mitochondria were decreased during pressure overload in rats. Moreover, anti-NOS1 antibody-sensitive protein was detected in cardiac mitochondria. These observations suggest the possibility that NOS uncoupling by a decrease in BH4 levels in cardiac mitochondria is implicated, at least in part, in the development of cardiac fibrosis leading to cardiac dysfunction induced by pressure overload.

\section{Acknowledgements}

The gift of BH4 from Daiichi Sankyo Co. (Tokyo, Japan) is gratefully acknowledged. This study was partially supported by a Private University High Technology Research Center Project matching fund subsidy from MEXT.

\section{References}

1. MacCarthy PA, Grieve DJ, Li JM, Dunster C, Kelly FJ and Shah AM: Impaired endothelial regulation of ventricular relaxation in cardiac hypertrophy: role of reactive oxygen species and NADPH oxidase. Circulation 104: 2967-2974, 2001.

2. Takimoto E and Kass DA: Role of oxidative stress in cardiac hypertrophy and remodeling. Hypertension 49: 241-248, 2007.

3. Kwon NS, Nathan CF and Stuehr DJ: Reduced biopterin as a cofactor in the generation of nitrogen oxides by murine macrophages. J Biol Chem 264: 20496-20501, 1989.

4. Tayeh MA and Marletta MA: Macrophage oxidation of L-arginine to nitric oxide, nitrite, and nitrate. Tetrahydrobiopterin is required as a cofactor. J Biol Chem 264: 19654-19658, 1989.

5. Werner-Felmayer G, Werner ER, Fuchs D, Hausen A, Reibnegger $\mathrm{G}$ and Wachter $\mathrm{H}$ : Tetrahydrobiopterin-dependent formation of nitrite and nitrate in murine fibroblasts. J Exp Med 172: 1599-1607, 1990.

6. Schmidt K, Werner ER, Mayer B, Wachter H and Kukovetz WR: Tetrahydrobiopterin-dependent formation of endotheliumderived relaxing factor (nitric oxide) in aortic endothelial cells. Biochem J 281: 297-300, 1992.

7. Werner ER, Gorren ACF, Heller R, Werner-Felmayer G and Mayer B: Tetrahydrobiopterin and nitric oxide: mechanistic and pharmacological aspects. Exp Biol Med 228: 1291-1302, 2003.

8. Wever RM, van Dam T, van Rijn HJ, de Groot F and Rabelink TJ: Tetrahydrobiopterin regulates superoxide and nitric oxide generation by recombinant endothelial nitric oxide synthase. Biochem Biophys Res Commun 237: 340-344, 1997.

9. Vásquez-Vivar J, Kalyanaraman B, Martásek P, Hogg N, Masters BS, Karoui H, Tordo P and Pritchard KA Jr: Superoxide generation by endothelial nitric oxide synthase: the influence of cofactors. Proc Natl Acad Sci USA 95: 9220-9225, 1998.

10. Xia Y, Tsai AL, Berka V and Zweier JL: Superoxide generation from endothelial nitric-oxide synthase. $\mathrm{A} \mathrm{Ca}^{2+} / \mathrm{calmodulin-}^{-}$ dependent and tetrahydrobiopterin regulatory process. J Biol Chem 273: 25804-25808, 1998.

11. Malo O, Desjardins F, Tanguay J, Tardif J, Carrier M and Perrault LP: Tetrahydrobiopterin and antioxidants reverse the coronary endothelial dysfunction associated with left ventricular hypertrophy in a porcine model. Cardiovasc Res 59: 501-511, 2003.

12. Moens AL, Takimoto E, Tocchetti CG, Chakir K, Bedja D, Cormaci G, Ketner EA, Majmudar M, Gabrielson K, Halushka MK, Mitchell JB, Biswal S, Channon KM, Wolin MS, Alp NJ, Paolocci N, Champion HC and Kass DA: Reversal of cardiac hypertrophy and fibrosis from pressure overload by tetrahydrobiopterin: efficacy of recoupling nitric oxide synthase as a therapeutic strategy. Circulation 117: 2626-2636, 2008.
13. Silberman GA, Fan THM, Liu H, Jiao Z, Xiao HD, Lovelock JD, Boulden BM, Widder J, Fredd S, Bernstein KE, Wolska BM, Dikalov S, Harrison DG and Dudley SC Jr: Uncoupled cardiac nitric oxide synthase mediates diastolic dysfunction. Circulation 121: 519-528, 2010.

14. Moens AL, Ketner EA, Takimoto E, Schmidt TS, O'Neill CA, Wolin MS, Alp NJ, Channon KM and Kass DA: Bi-modal dose-dependent cardiac response to tetrahydrobiopterin in pressure-overload induced hypertrophy and heart failure. J Mol Cell Cardiol 51: 564-569, 2011.

15. Takimoto E, Champion HC, Li M, Ren S, Rodriguez ER, Tavazzi B, Lazzarino G, Paolocci N, Gabrielson KL, Wang Y and Kass DA: Oxidant stress from nitric oxide synthase-3 uncoupling stimulates cardiac pathologic remodeling from chronic pressure load. J Clin Invest 115: 1221-1231, 2005.

16. Brookes PS: Mitochondrial nitric oxide synthase. Mitochondrion 3: 187-204, 2004.

17. Haynes V, Elfering S, Traaseth $\mathrm{N}$ and Giulivi C: Mitochondrial nitric-oxide synthase: enzyme expression, characterization, and regulation. J Bioenerg Biomembr 36: 341-346, 2004.

18. Finocchietto PV, Franco MC, Holod S, Gonzalez AS, Converso DP, Arciuch VGA, Serra MP, Poderoso JJ and Carreras MC: Mitochondrial nitric oxide synthase: a masterpiece of metabolic adaptation, cell growth, transformation, and death. Exp Biol Med 234: 1020-1028, 2009.

19. Kanai AJ, Pearce LL, Clemens PR, Birder LA, VanBibber MM, Choi SY, de Groat WC and Peterson J: Identification of a neuronal nitric oxide synthase in isolated cardiac mitochondria using electrochemical detection. Proc Natl Acad Sci USA 98: 14126-14131, 2001

20. Brown GC and Borutaite V: Nitric oxide and mitochondrial respiration in the heart. Cardiovasc Res 75: 283-290, 2007.

21. Carreras MC, Franco MC, Finocchietto PV, Converso DP, Antico Arciuch VG, Holod S, Peralta JG and Poderoso JJ: The biological significance of mtNOS modulation. Front Biosci 12: 1041-1048, 2007.

22. Dedkova EN and Blatter LA: Characteristics and function of cardiac mitochondria nitric oxide synthase. J Physiol 587: 851-872, 2009.

23. Faerber G, Barreto-Perreia F, Schoepe M, Gilsbach R, Schrepper A, Schwarzer M, Mohr FW, Hein L and Doenst T: Induction of heart failure by minimally invasive aortic constriction in mice: reduced peroxisome proliferator-activated receptor $\gamma$ coactivator levels and mitochondrial dysfunction. J Thorac Cardiovasc Surg 141: 492-500, 2011.

24. Givvimani S, Munjal C, Tyagi N, Sen U, Metreveli N and Tyagi SC: Mitochondrial division/mitophagy inhibitor (Mdivi) ameliorates pressure overload induced heart failure. PLoS One 7: e32388, 2012.

25. Dai DF, Hsieh EJ, Liu Y, Chen T, Beyer RP, Chin MT, MacCoss MJ and Rabinovitch PS: Mitochondrial proteome remodelling in pressure overload-induced heart failure: the role of mitochondrial oxidative stress. Cardiovasc Res 93: 79-88, 2012.

26. Bugger H, Schwarzer M, Chen D, Schrepper A, Amorim PA, Schoepe M, Nguyen TD, Mohr FW, Khalimonchuk O, Weimer BC and Doenst T: Proteomic remodelling of mitochondrial oxidative pathways in pressure overload-induced heart failure. Cardiovasc Res 85: 376-384, 2010.

27. Yorikane R, Sakai S, Miyauchi T, Sakurai T, Sugishita Y and Goto K: Increased production of endothelin-1 in the hypertrophied rat heart due to pressure overload. FEBS Lett 332: 31-34, 1993.

28. Heller R, Unbehaun A, Schellenberg B, Mayer B, WernerFelmayer G and Werner ER: L-ascorbic acid potentiates endothelial nitric oxide synthesis via a chemical stabilization of tetrahydrobiopterin. J Biol Chem 276: 40-47, 2001.

29. Valent S and Tóth M: Spectrophotomeric analysis of the protective effect of ascorbate against spontaneous oxidation of tetrahydrobiopterin in aqueous solution: kinetic characteristics and potentiation by catalase of ascorbate action. Int $\mathbf{J}$ Biochem Cell Biol 36: 1266-1280, 2004.

30. Tóth M, Kukor Z and Valent S: Chemical stabilization of tetrahydrobiopterin by L-ascorbic acid: contribution to placental endothelial nitric oxide synthase activity. Mol Hum Reprod 8: 271-280, 2002.

31. Fellet AL, Balaszczuk AM, Arranz C, López-Costa JJ, Boveris A and Bustamante J: Autonomic regulation of pacemaker activity: role of heart nitric oxide synthases. Am J Physiol Heart Circ Physiol 291: H1246-H1254, 2006. 
32. Wajima T, Shimizu S, Hiroi T, Ishii M and Kiuchi Y: Reduction of myocardial infarct size by tetrahydrobiopterin: possible involvement of mitochondrial $\mathrm{K}_{\mathrm{ATP}}$ channels activation through nitric oxide production. J Cardiovasc Pharmacol 47: 243-249, 2006.

33. Hiroi T, Wajima T, Kaneko Y, Kiuchi Y and Shimizu S: An important role of increase in tetrahydrobiopterin via $\mathrm{H}_{2} \mathrm{O}_{2}$-JAK2 signalling pathway in late phase of ischaemic preconditioning. Exp Physiol 95: 609-621, 2010.

34. Shimizu S, Shiota K, Yamamoto S, Miyasaka Y, Ishii M, Watabe T, Nishida M, Mori Y, Yamamoto T and Kiuchi Y: Hydrogen peroxide stimulates tetrahydrobiopterin synthesis through the induction of GTP-cyclohydrolase I and increases nitric oxide synthase activity in vascular endothelial cells. Free Radic Biol Med 34: 1343-1352, 2003.

35. Zaobornyj T, Valdez LB, La Padula P, Costa LE and Boveris A Effect of sustained hypobaric hypoxia during maturation and aging on rat myocardium. II. mitNOS activity. J Appl Physiol 98: 2370-2375, 2005.
36. Giulivi C, Kato K and Cooper CE: Nitric oxide regulation of mitochondrial oxygen consumption I: cellular physiology. Am J Physiol Cell Physiol 291: C1225-C1231, 2006.

37. Baines CP: The cardiac mitochondrion: nexus of stress. Annu Rev Physiol 72: 61-80, 2010.

38. Konstantinidis K, Whelan RS and Kitsis RN: Mechanisms of cell death in heart disease. Arterioscler Thromb Vasc Biol 32: $1552-1562,2012$

39. García-Cardeña G, Oh P, Liu J, Schnitzer JE and Sessa WC: Targeting of nitric oxide synthase to endothelial cell caveolae via palmitoylation: implications for nitric oxide signaling. Proc Natl Acad Sci USA 93: 6448-6453, 1996.

40. Feron $\mathrm{O}$ and Balligand JL: Caveolins and the regulation of endothelial nitric oxide synthase in the heart. Cardiovasc Res 69: 788-797, 2006 